\title{
Fluid Resuscitation for Septic Shock - A 50-Year Perspective: From Dogma to Skepticism
}

\author{
Robert Raschke, MD \\ Arooj Kayani, MD \\ Samir Sultan, DO \\ Stephanie Fountain, MD \\ Moustafa Abidali, DO \\ Kyle Henry, MD
}

\begin{abstract}
Banner University Medical Center Phoenix Phoenix, AZ USA
\end{abstract}

Few clinicians would challenge the contention that fluid resuscitation of sepsis improves tissue perfusion thereby protecting end-organs from injury. This is an underlying tenet of current Surviving Sepsis Campaign (SSC) recommendations (1) and Center for Medicare and Medicaid Services (CMS) mandate that hospitals report sepsis bundle compliance as a measure of healthcare quality. It has persisted for decades despite the lack of convincing empirical evidence that fluid resuscitation improves clinical outcomes. To the contrary, large randomized controlled trials have shown that aggressive intravenous fluid resuscitation prolongs the need for mechanical ventilation (2) and increases mortality in some patients (3) - more on these studies later. Furthermore, the pathophysiological rationale commonly used to explain why fluid resuscitation ought to be beneficial has been challenged by a growing body of evidence. This article started as a journal club held by our Pulmonary Critical Care fellows, but we expanded the scope to review other related studies over the past 50 years that challenge the current accepted paradigm of aggressive fluid resuscitation of sepsis and septic shock.

The positive results of River's early goal-directed therapy (EGDT) trial in the early 2000s (4) were inexplicable to many that followed previous literature. EGDT required aggressive fluid resuscitation to achieve a central venous pressure (CVP) $\geq 8-12$ $\mathrm{cmH}_{2} \mathrm{O}$, culminating in a mean positive fluid balance $>13 \mathrm{~L}$ at 72 hours. But it had been recognized for decades that CVP could not reasonably be used in this manner. In 1965, Dr. Max Weil (considered by some the founder of critical care medicine) made the observation that the CVP is primarily an index of right ventricular function rather than an index of volume status (5). The widely-held concept (which has persisted since 1965) that low venous pressure indicates low blood volume was developed using data from normal subjects and was not valid in critical illness. Elevated CVP reflects incompetence of the heart to accept the blood returned to it. As such, CVP ought to be used primarily to limit over-resuscitation rather than to indicate when more fluids are needed (5).

These early observations and decades of corroborating evidence were set-aside for yet another decade as EGDT was systematically endorsed. Near the peak of enthusiasm for EGDT, a meta-analysis of 24 studies demonstrated no significant relationship between CVP and blood volume $\left(r^{2}=0.02\right)$ or fluid responsiveness $\left(r^{2}=0.03\right)(6)$. A graph 
from that article based on 1500 simultaneous measurements of CVP and blood volume graphically illustrates the apparent lack of any association, supporting Dr Weil's clinical observations from over 40 years earlier (Figure 1).

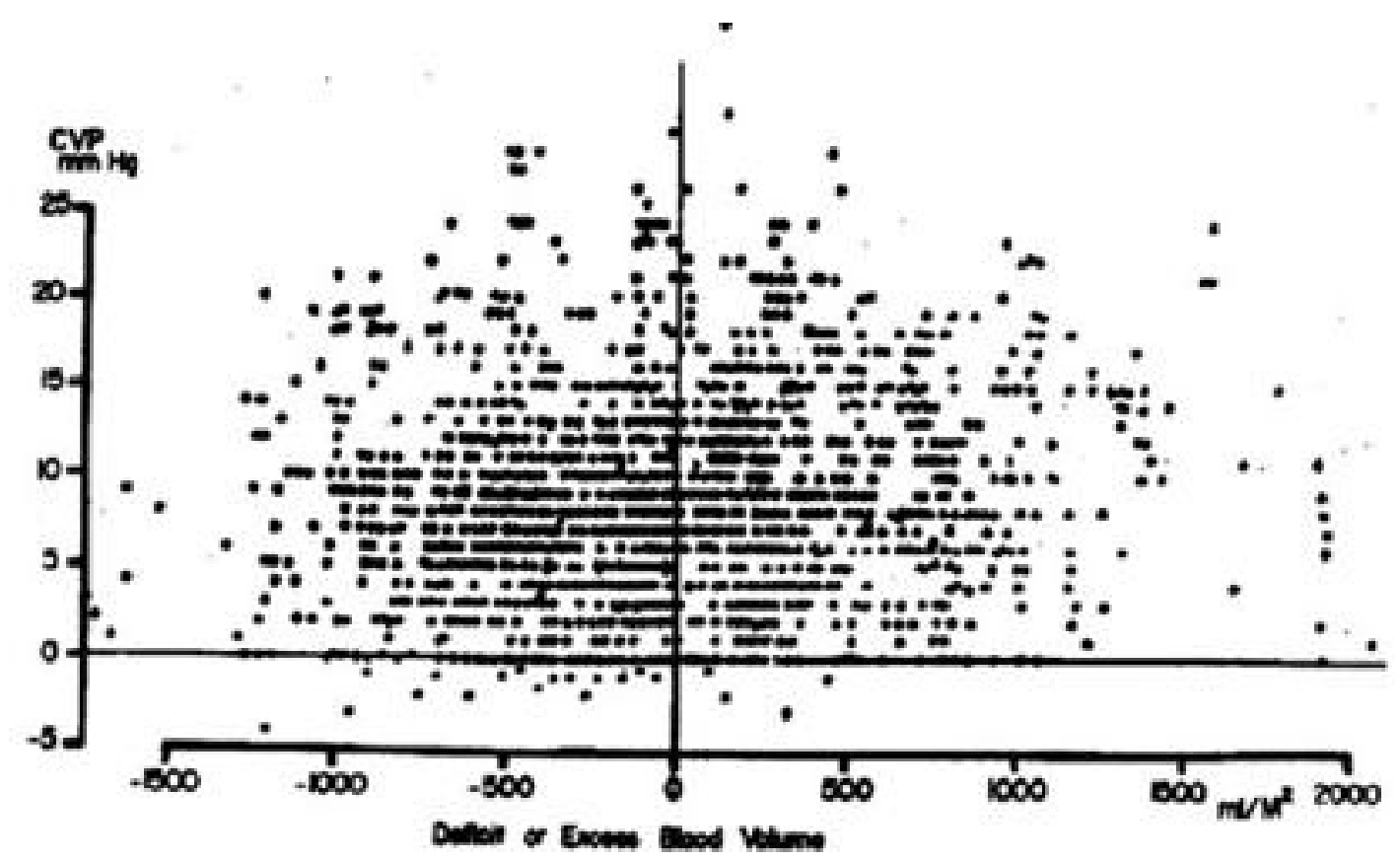

Figure 1. Graph of simultaneous measurements of blood volume and central venous pressure (CVP) in a heterogenous cohort of $188 \mathrm{ICU}$ patients demonstrating no association between these two variables $(r=0.27)(6)$.

Nevertheless, EGDT was avidly endorsed by authoritarian professional organizations and immense time and effort expended on national and international efforts to promote it's systematic implementation. Several observational studies showed that systematic implementation of EGDT in healthcare institutions decreased sepsis mortality $(7,8)$. However, the use of historical controls in these studies allowed other simultaneous changes in ICU practice and the Hawthorne effect to potentially confound their results.

In 2006, the ARDS clinical trials network published a multi-center controlled trial that randomized 1000 patients with acute lung injury to liberal or conservative fluid management (2). Approximately $70 \%$ of the patients in the study satisfied current criteria for sepsis (were classified as having sepsis or pneumonia with acute organ system dysfunction). Critical appraisal of the study revealed that $>90 \%$ of screened patients were excluded, complicated fluid management protocols were unlikely to be practical for routine use and the study was not blinded. But the study methodology was otherwise essentially sound. Liberal fluid management achieved a more positive fluid balance over the first 7 days $(+6992+/-502 \mathrm{~mL}$ vs. $-136+/-491 \mathrm{~mL} p<0.001)$, but failed to reduce the incidence of shock or acute renal failure requiring dialysis. It was instead associated with significantly prolonged ventilator dependence (12.1 vs. 14.6 ventilatorfree days, $p<0.001$ ) and prolonged ICU length-of-stay (11.2 vs. $13.4 \mathrm{ICU}$-free days, $p<0.001)$. These results seemed contrary to those of Rivers and we struggled at the 
time to reconcile the two. Our shared impression at journal club is that aggressive fluid resuscitation followed by permissive hypervolemia, such as seen in the liberal fluid management arm of this study, is still common in current practice. This study suggests that this approach significantly prolongs recovery from acute lung injury.

Maitland's study of fluid boluses in African children in 2011 is remarkable as the only large prospective randomized controlled trial (RCT) to study the clinical effect of early fluid resuscitation in patients with severe infections (3). The study randomized children with high fever and clinical evidence of impaired perfusion to three groups: $5 \%$ albumin bolus, normal saline bolus or no bolus. The safety monitoring committee ended the study after 3141 of 3600 projected patients had been enrolled, based on evidence that administration of either type of fluid bolus significantly increased mortality (RR 1.45 $95 \% \mathrm{Cl}: 1.13-1.86 \mathrm{p}=0.003)$. Methodology was limited by available healthcare infrastructure. Although the proportion of patients with sepsis cannot be calculated, 39\% had a lactate $>5 \mathrm{mmol} / \mathrm{L}$. The study had reasonable internal validity, but significant challenges to external validity - the mean patient age was 23 months, and $57 \%$ had malaria. However, the authors noted: "The excess mortality with fluid resuscitation was consistent across all subgroups, irrespective of physiological derangement (whether or not the patient was in shock) or underlying microbial pathogen, raising fundamental questions about our understanding of the pathophysiology of critical illness." The authors speculated that the neuro-hormonal vasoconstrictor response to shock might confer protection by reducing perfusion to non-vital tissues and that rapid reversal with fluid resuscitation could therefore be harmful. This specific hypothesis was supported by a post-hoc analysis that showed that the increased mortality associated with fluid boluses could not be explained by an increase in pulmonary or cerebral edema. Although the generalizability of this study is limited, there is no comparable RCT of fluid boluses in any other group of patients to refute it's findings.

The review of resuscitation fluids by Myberg and Mythen in 2013 (9) emphasized ongoing uncertainty and reasoned against a protocolized approach driving aggressive fluid resuscitation stating "the requirements for and response to fluid resuscitation vary greatly during the course of any critical illness. No single physiological or biochemical measurement adequately reflects the complexity of fluid depletion or the response to fluid resuscitation." They reviewed observational evidence that the development of positive fluid balance and elevated CVP were associated with increased mortality in patients with sepsis. They pointed out that intravenous fluids should be considered as a drug with potentially serious side effects: interstitial edema - and in the case of normal saline, hyperchloremic acidosis and acute kidney injury. They recommended modest amounts of balanced isotonic salt solutions guided by clinical consideration of multiple individual patient factors, cautioned against continuing fluid resuscitation after the first 24 hours of illness and encouraged early initiation of norepinephrine.

Myberg's review was published about the time that the results of three randomized controlled trials, which cumulatively enrolled 4201 patients at 138 emergency departments and ICUs internationally conclusively refuted any clinical benefit of EGDT (10-12). Shortly thereafter, CMS paradoxically mandated monthly sepsis bundle 
compliance reporting as a measure of healthcare quality, strongly incentivizing hospitals to systematically institute sepsis bundles, even though they had just been proven to be ineffective.

We greatly enjoyed the review of fluid therapy in sepsis by Marik and Bellomo (13). They argue that the standard pathophysiological explanation for the theoretical benefit of fluid resuscitation in sepsis is contradicted by a growing body of evidence. Septic shock is not characterized by hypovolemia but rather by vasoplegia and injury to the endothelial glycocalyx. Resultant microvascular permeability and propensity to interstitial edema impairs organ function. As such, restoration of vascular tone (including that of capacitance veins) is the preferred initial intervention to restore perfusion. Elevating the CVP $>8 \mathrm{cmH}_{2} \mathrm{O}$ with fluid boluses does not reliably improve preload and cardiac output as commonly supposed. Instead, it most often overfills the heart, inducing acute diastolic dysfunction in a majority of patients. This paradoxically reduces stroke volume and moves the patient onto the flat portion of the Frank Starling curve mitigating any potential augmentation of cardiac fluid by further fluid administration. Elevated CVPs in this setting are not an indication of successful fluid resuscitation but rather a sign of cardiac incompetence to accommodate iatrogenic hypervolemia. Cardiac natriuretic peptides released in response to cardiac overfilling cleave glycoproteins that make up the endothelial glycocalyx further injuring it. Venous backpressure worsens organ perfusion and increases interstitial edema, particularly affecting the kidneys. However, cellular hypoxia and bioenergetics failure does not occur and is not the cause of lactic acidosis in septic shock as is often supposed. Elevated lactate levels are instead caused by bioenergetic-coupling of epinephrine-induced stimulation of $\mathrm{Na} / \mathrm{K}$ ATPase activity to aerobic glycolysis. The critical level of oxygen delivery below which oxygen consumption falls is almost never associated with septic shock, and increasing oxygen delivery has been not been shown to improve oxygen consumption or lower lactate levels. Attempts to specifically increase oxygen delivery in sepsis have in fact worsened survival.

Furthermore, only a minority of patients with sepsis respond with increased stroke volume after a fluid bolus. Hemodynamic improvements seen in "fluid responders" return to baseline within an hour. 95\% of administered fluid is rapidly sequestered in tissues where it contributes to organ dysfunction. Goal-directed fluid administration achieves only a transient hemodynamic improvement in a minority of patients at the cost of accumulating injurious tissue edema in all. Analysis of five serial randomized controlled trials that ultimately disproved the efficacy of EGDT shows that sepsis mortality has been fallen significantly over the past 15 years in association with a tendency towards significantly more conservative fluid management (approx. 13L/72hrs vs. $6 \mathrm{~L} / 72$ hours) suggesting that a more conservative approach to fluid resuscitation may explain improved survival (Figure 2). 


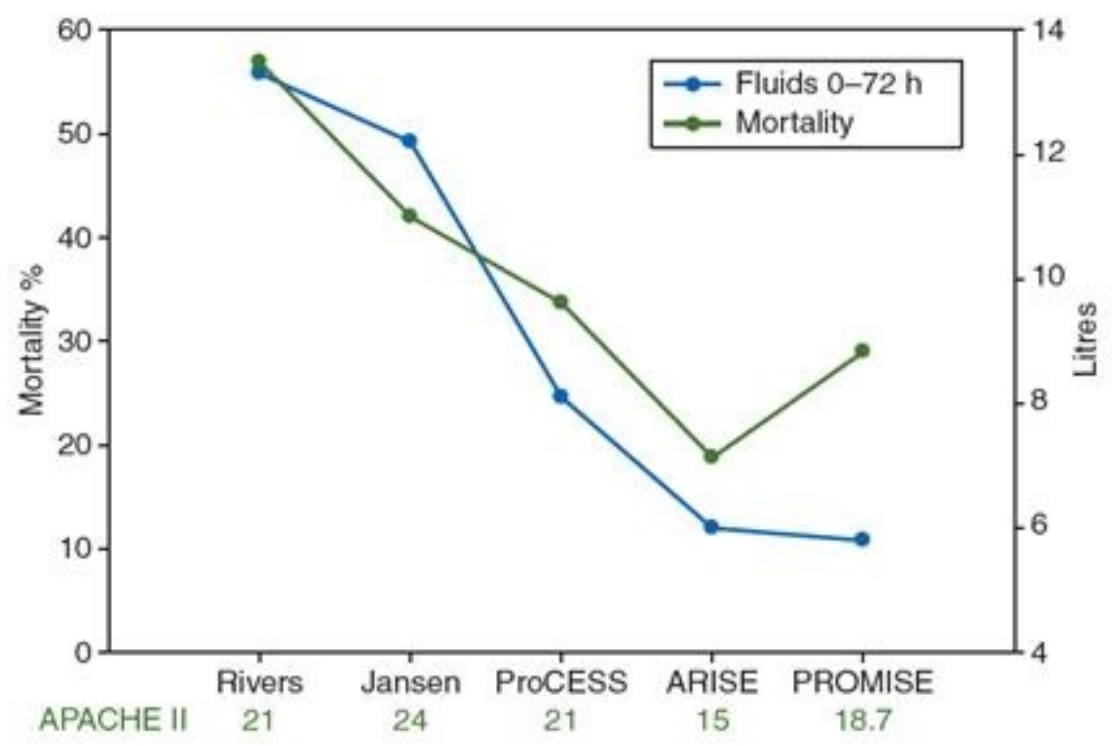

Figure 2. Fluid administered between enrolment and $72 \mathrm{~h}$ and 90-day mortality in the control arm of the Early Goal Directed Therapy (EGDT) Studies performed between 2001 and 2015. APACHE II=APACHE II Severity of illness scoring system (0-71) (13).

Marik and Bellomo (13) recommend early administration of norepinephrine, which can be safely administered via a well-functioning peripheral intravenous catheter and cautious administration of small volume fluid boluses $(200-500 \mathrm{~mL})$ only in patients in whom passive leg raise (a reversible fluid bolus) can be demonstrated to augment stroke volume. They argue that CVP, central venous oxygen saturation and lactate should not be used to guide fluid management, and should in fact not even be measured.

Taken individually, each of these studies seems anomalous in the context of our preconceived notion that aggressive fluid resuscitation must be beneficial. Taken together, they comprise a cohesive argument that ought to change our bedside care.

There certainly isn't any convincing or enduring empirical evidence that aggressive fluid resuscitation of sepsis is clinically beneficial. There is only flawed pathophysiologic rationale and dogma. The common practice of aggressive fluid resuscitation followed by prolonged permissive hypervolemia should be actively avoided. As we struggle to comply with a CMS mandate regarding sepsis bundle compliance in the face of overwhelming evidence that it doesn't work, we recommend a focus on early administration of appropriate antibiotics and maintenance of adequate perfusion pressure with vasopressors - the only bundle components likely to be associated with improved patient outcomes.

\section{References}

1. Dellinger RP, Levy MM, Rhodes A, et al. ; Surviving Sepsis Campaign Guidelines Committee including The Pediatric Subgroup. Surviving Sepsis Campaign: 
international guidelines for management of severe sepsis and septic shock, 2012. Intensive Care Med. 2013 Feb;39(2):165-228. [CrossRef] [PubMed]

2. National Heart, Lung, and Blood Institute Acute Respiratory Distress Syndrome (ARDS) Clinical Trials Network, Wiedemann HP, Wheeler AP, Bernard GR, et al. Comparison of two fluid-management strategies in acute lung injury. N Engl J Med. 2006 Jun 15;354(24):2564-75. [CrossRef] [PubMed]

3. Maitland K, Kiguli S, Opoka RO, et al. Mortality after fluid bolus in African children with severe infection. N Engl J Med. 2011 Jun 30;364(26):2483-95. [CrossRef] [PubMed]

4. Rivers E, Nguyen B, Havstad S, et al. Early goal-directed therapy in the treatment of severe sepsis and septic shock. N Engl J Med. 2001 Nov 8;345(19):1368-77. [CrossRef] [PubMed]

5. Weil MH, Shubin H, Rosoff L. Fluid repletion in circulatory shock. JAMA. 1965;192:84-90. [CrossRef] [PubMed]

6. Marik PE, Baram M, Vahid B. Does central venous pressure predict fluid responsiveness? A systematic review of the literature and the tale of seven mares. Chest. 2008 Jul;134(1):172-8. [CrossRef] [PubMed]

7. Ferrer R, Artigas A, Levy MM, et al. Improvement in process of care and outcome after a multicenter severe sepsis educational program in Spain. JAMA. 2008 May 21;299(19):2294-303. [CrossRef] [PubMed]

8. Rhodes A, Phillips G, Beale R, et al. The Surviving Sepsis Campaign bundles and outcome: results from the International Multicentre Prevalence Study on Sepsis (the IMPreSS study). Intensive Care Med. 2015 Sep;41(9):1620-8. [CrossRef] [PubMed]

9. Myburgh JA, Mythen MG. Resuscitation fluids. N Engl J Med. 2013 Sep 26;369(13):1243-51. [CrossRef] [PubMed]

10. ProCESS Investigators, Yealy DM, Kellum JA, Huang DT, et al. A randomized trial of protocol-based care for early septic shock. N Engl J Med. 2014 May 1;370(18):168393. [CrossRef] [PubMed]

11. ARISE Investigators; ANZICS Clinical Trials Group, Peake SL, Delaney A, Bailey M, et al. Goal-directed resuscitation for patients with early septic shock. N Engl J Med. 2014 Oct 16;371(16):1496-506. [CrossRef] [PubMed]

12. Mouncey PR, Osborn TM, Power GS, et al. Trial of early, goal-directed resuscitation for septic shock. N Engl J Med. 2015 Apr 2;372(14):1301-11. [CrossRef] [PubMed]

13. Marik P, Bellomo R. A rational approach to fluid therapy in sepsis. Br J Anaesth. 2016 Mar;116(3):339-49. [CrossRef] [PubMed] 